\title{
An Insight to Oral Epithelial Dysplasia
}

\author{
Varun Rastogi, Naveen Puri, Satyaranjan Mishra, Swati Arora, Geetpriya Kaur, Lalita Yadav
}

\begin{abstract}
Oral dysplasia is a potentially precancerous lesion diagnosed histologically. While the risk of progression is associated with histological grade, it is currently impossible to predict accurately which lesions will progress. Although most oral pathologists recognize and accept the criteria for grading epithelial dysplasia based on architectural and cytological changes, there can be considerable interexaminer and intraexaminer variation in the assessment of the presence or absence and the grade of oral epithelial dysplasia. This article reviews the alterations, criteria, different grading systems and the markers used for assessing the malignant transformation of epithelial dysplasia.
\end{abstract}

Keywords: Epithelial dysplasia, Atypia, Genetic, Epigenetic, Grading, Biomarkers.

How to cite this article: Rastogi V, Puri N, Mishra S, Arora S, Kaur G, Yadav L. An Insight to Oral Epithelial Dysplasia. Int J Head Neck Surg 2013;4(2):74-82.

\section{Source of support: Nil}

\section{Conflict of interest: None}

\section{INTRODUCTION}

The term 'Dysplasia' was introduced by Reagon 1958 in relation to the cells exfoliated from lesions of the uterine cervix. Dysplasia is an ominous premalignant change. In past, epithelial dysplasia, epithelial atypia and dyskeratosis were used synonymously. The first change suggesting malignant transformation is dysplasia. Dysplasia (dys = abnormal $/$ bad; plasia $=$ growth) is defined as 'A precancerous lesion of stratified squamous epithelium characterized by cellular atypia and loss of normal maturation and stratification short of carcinoma in situ'. Pindborg (1977) defined epithelial dysplasia as the term used for 'A lesion in which part of the thickness of the epithelium is replaced by the cells showing varying degree of cellular atypia' . ${ }^{1}$ Lumermann et al (1995) defined epithelial dysplasia as 'A diagnostic term used to describe the histopathological changes seen in chronic progressive and premalignant disorders of oral mucosa' ${ }^{2}$

The presence of dysplastic areas in the epithelium of the upper aerodigestive tract is believed to be associated with a likely progression to cancer. Dysplastic features of a stratified squamous epithelium are characterized by cellular atypia and loss of normal maturation and stratification. ${ }^{1}$ There is support for the view that the more severe the dysplasia the greater the likelihood is of progression to malignancy. ${ }^{3}$ It has been demonstrated that the accumulation of genetic and epigenetic alteration takes place during malignant development and in the oral mucosa this is reflected by a series of well-defined clinical and histological changes depicting dysplasia. ${ }^{4}$ The histological findings of dysplasia therefore indicate no more than a lesion has a statistically increased risk of malignant change, but cannot be used for confident prediction of malignant change in any individual case. Clearly, studies of potential biomarkers are needed in order to introduce more objectivity. $^{5}$

\section{ALTERATIONS IN DYSPLASIA}

Dysplasia refers to a series of subtle changes in cells signifying that anaplasia will develop soon. Dysplasia is theoretically reversible and therefore not yet malignant. Dysplasia is a premalignant change. It is a change at tissue level while atypia is a change at cellular level. Dysplasia is reversible and therefore a controlled cellular alteration. When the underlying inciting stimulus is removed, the dysplastic alterations revert to normal.

The alteration in dysplasia includes genetic changes, epigenetic changes and surface alterations. The sum total of these physical and morphological alterations are of diagnostic and prognostic relevance and are designated as precancerous changes.

A genetic change involves complex process due to the interaction of the host (genetic factor) with carcinogens in the environment and includes activation of proto-oncogenes, inactivation of the tumor suppressor genes and inactivation of the genomic stability genes.

Epigenetic refers to heritable changes in the gene expression that occur without alteration in DNA sequence. CH Waddington in 1942 defined it as 'The branch of biology which studies the causal interactions between genes and their products which bring the phenotype into being'. ${ }^{6}$ Chemical modifications to DNA and its associated proteins can alter gene expression without altering the DNA sequence whereas the genetic aberrations change the expression by altering the sequence of $\mathrm{A}-\mathrm{T}$ and $\mathrm{C}-\mathrm{G}$.

Epigenetic changes involve modifications in the activation of certain genes, but not the basic structure ofDNA. Additionally, the chromatin proteins associated with DNA may be activated or silenced. This accounts for why the differentiated cells in a multicellular organism express only the genes that are necessary for their own activity. Epigenetic changes are preserved when cells divide. Most epigenetic changes only occur within the course of one individual organism's lifetime, but some epigenetic changes are inherited from one generation to the next. ${ }^{6,7}$ 
Two primary and interconnected epigenetic mechanisms include DNA methylation and modification of histones. Also RNA is intimately involved in the formation of a repressive chromatin state.

The surface alteration includes cellular adaptations, reversible and irreversible changes. The reversible changes are reversible if causative factors are removed. If they persist, dysplastic cells escapes normal homeostatic control and assume the autonomy of tumor cells. The irreversible changes is characterized by accelerated cell division, which facilitates accumulation of genetic damage and further drives toward path of transformation and lead to cell death or neoplastic transformation.

\section{CRITERIA FOR DYSPLASIA}

When architectural disturbance is accompanied by cytological atypia (variations in the size and shape of the keratinocytes) the term dysplasia applies.

Criteria used for diagnosing oral epithelial dysplasia ${ }^{3}$ are listed in Table 1 . These features could be broadly categorized as changes to the architecture (strata) of the epithelium and those that manifest as cellular atypia (Figs 1 to 9 ). ${ }^{3}$

\section{GRADING OF DYSPLASIA}

Many dysplastic features in varying combinations have been used for grading. However, difficulties have been encountered in assessing and standardizing the different degrees of epithelial dysplasia. Many systems of grading epithelial dysplasia have been proposed in order to standardize the severity of dysplastic features. In addition, the parameters considered in the histological assessment should be biologically meaningful, reflecting the malignant potential of the lesion. ${ }^{8}$ The various grading systems put forth by different authors are as follows:

1. Smith and Pindborg photograhic method (1969)

2. Mehta et al (1971)

3. Bancozy and Csiba (1976)

4. WHO (1978)

5. Kramer (1980)

6. Burkhardt and Maerkar (1981)

7. Shafer (1983)

8. Lumermann $\mathrm{H}$ et al (1995)

9. Neville et al (1995)

10. Speight PM et al (1996)

11. Kuffer and Lombardi (2002)

12. Ljubljana (2003)

13. Brothwell DJ (2003)

14. WHO system (2005)

15. Binary system (2005)

\section{Smith and Pindborg Method}

A scoring system based on a set of photographic standards was suggested in the late 1960s (Smith and Pindborg 1969): Smith-Pindborg criteria ${ }^{9}$ is listed in Table 2. They described a simple system for assessing epithelial dysplasia to produce a numerical score or epithelial atypia index. Katzet al $(1985)^{10}$ found the system to be of considerable value for purposes of standardization and eliminated observer bias by the use of standard photographs. They evaluated 13 histological features which were standardized by a set of photographs. Each feature was graded 'absent', 'slight' and 'marked' as follows:

\section{Grading}

Epithelial dysplasia index is the sum of 13 scores. Each feature carries a weighted score like basal cell hyperplasia $=4$ and marked pleomorphism of cells and nuclei $=6$. A grading of 'none' was scored 0 (zero). Grading of 'slight' or 'marked' was scored from 1 to 10.

- The grading finally was done as follows:

\begin{tabular}{|c|c|}
\hline Total score (EDI) & Grade \\
\hline - $\quad 0-10$ & Not dysplastic \\
\hline - $11-25$ & Mild dysplasia \\
\hline - $\quad 26-45$ & Moderate dysplasia \\
\hline - $46-75$ & Severe dysplasia \\
\hline
\end{tabular}

The drawback is that the system relies on the weighting of the individual criteria originally made by the authors and, therefore does not solve the problem of subjectivity. The system is rather laborious and has not gained wide use for routine diagnostic purposes. Warnakulasuriya (2001) ${ }^{11}$ commented on this system and noted that even inflammatory or reactive lesions which are considered non-neoplastic may show some features of dysplasia.

\section{Mehta et al (1971)}

Mehta et al diagnosed epithelial dysplasia when two or more features of Smith-Pindborg criteria were present.

\section{Bancozy and Csiba (1976) ${ }^{12}$}

They diagnosed epithelial dysplasia using the following criteria:

- Irregular epithelial stratification

- Increased density of the basal cell layer or prickle cell layer or both

- Increased number of mitotic figures

- Increased nuclear cytoplasmic ratio

- Loss of polarity of cells

- Nuclear pleomorphism

- Hyperchromatism 


\begin{tabular}{ll}
\hline & Table 1: Criteria used for dysplasia \\
\hline Architectural/tissue changes & Cytological changes \\
\hline Irregular epithelial stratification & Abnormal variation in nuclear size (anisonucleosis) \\
Loss of polarity of basal cells & Abnormal variation in nuclear shape (nuclear pleomorphism) \\
Basal cell hyperplasia & Abnormal variation in cell size (anisocytosis) \\
Drop-shaped rete ridges & Abnormal variation in cell shape (cellular pleomorphism) \\
Increased number of mitotic figures & Increased nuclear-cytoplasmic ratio \\
Abnormally superficial mitoses & Increased nuclear size \\
Premature keratinization in single cells (dyskeratosis) & Atypical mitotic figures \\
Keratin pearls within rete ridges & Increased number and size of nucleoli \\
& Hyperchromasia \\
\hline
\end{tabular}

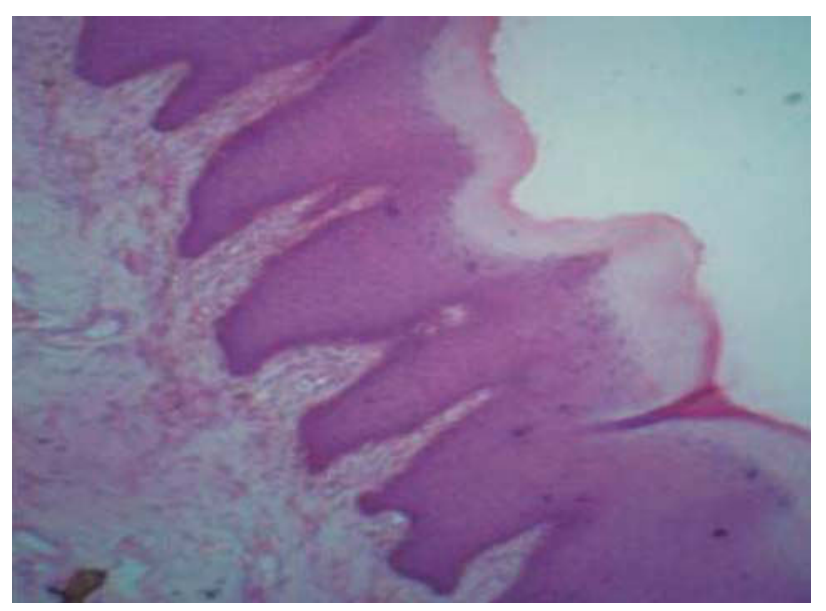

Fig. 1: Hyperkeratosis

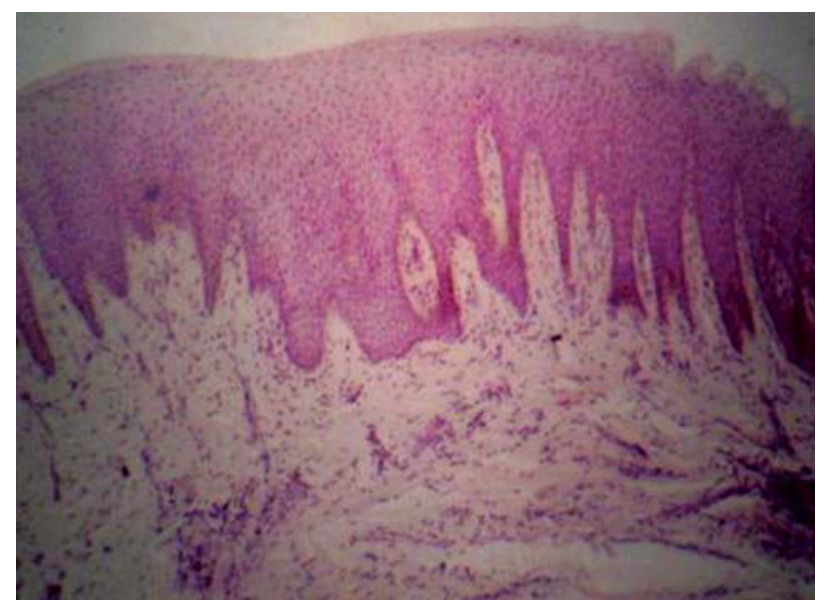

Fig. 2: Alteration of the rete pegs

- Keratinization of single cells or cell groups in the prickle cell layer

- Loss of intercellular adherence.

\section{Grading}

They graded epithelial dysplasia as:

- Mild: When two of the above listed histological changes were present.

- Moderate: When two to four changes were present.

- Severe: When five or more of the changes were present.

The drawback is that the grading was based on subjective interpretation of the features and did not take

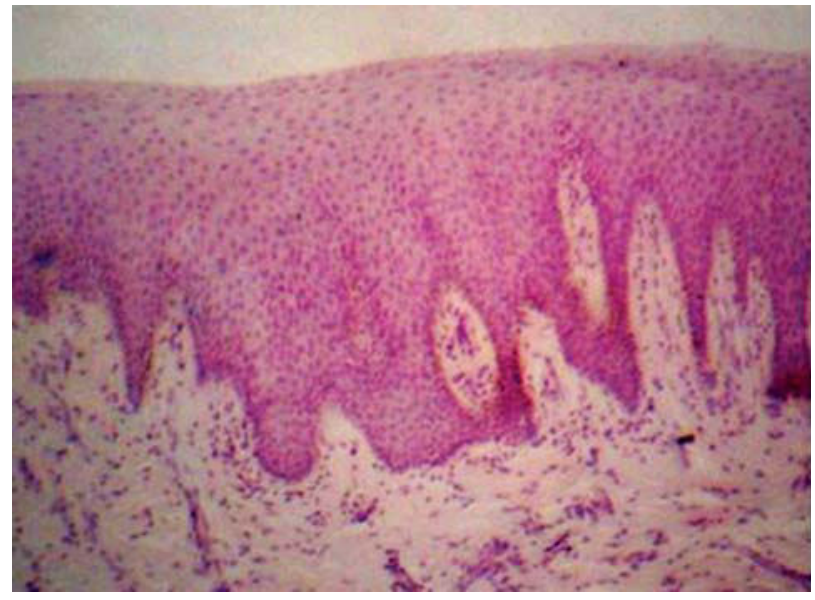

Fig. 3: Loss of stratification

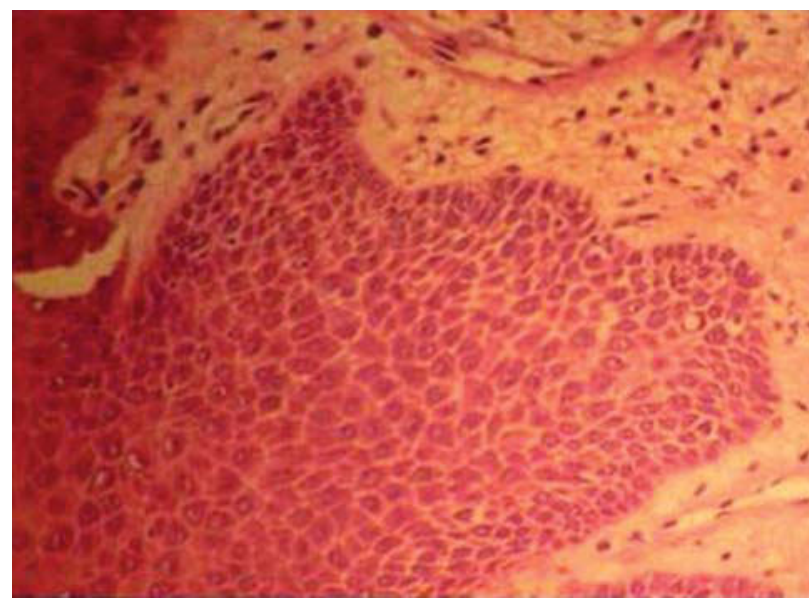

Fig. 4: Basal cell hyperplasia

into account which factor was important in determining the malignant potential.

\section{WHO System (1978)}

The 12 histological characteristics that characterized the epithelial dysplasia are:

- Loss of polarity of basal cells

- The presence of more than one layer of cells having basaloid appearance

- An increased nuclear-cytoplasmic ratio

- Drop-shaped rete pegs 


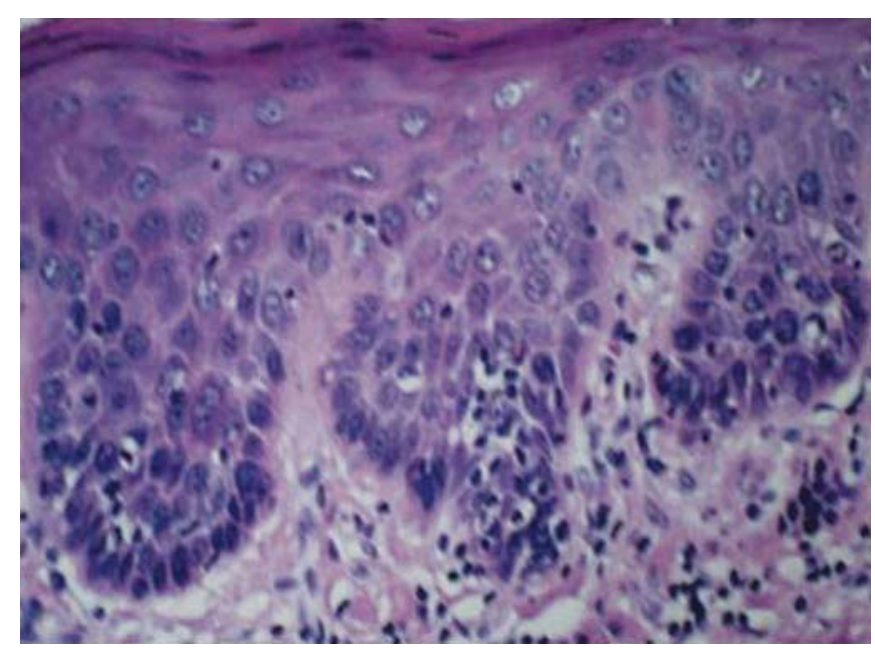

Fig. 5: Increased cellular density

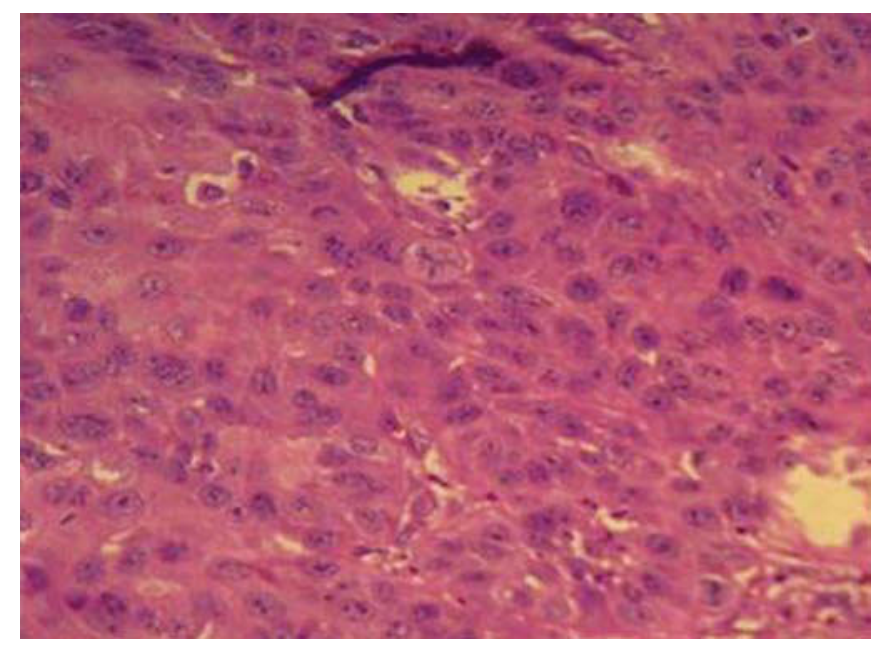

Fig. 6: Loss of polarity of basal cells

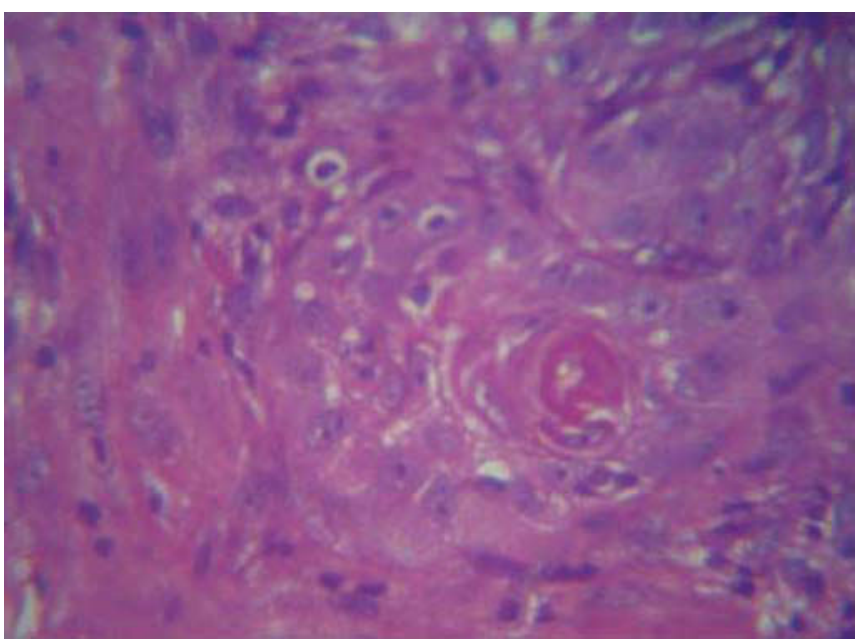

Fig. 7: Keratin pearls: Tight concentric rings of flattened keratinocytes

- Irregular epithelial stratification

- Increased number of mitotic figures

- The presence of mitotic figures in the superficial half of the epithelium

- Cellular polymorphism

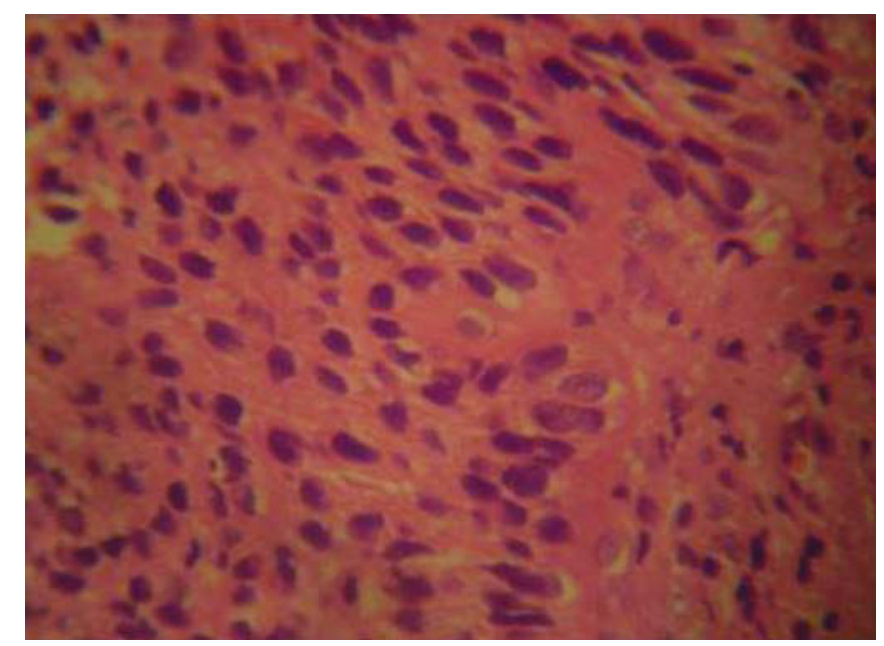

Fig. 8: Nuclear hyperchromatism and pleomorphism

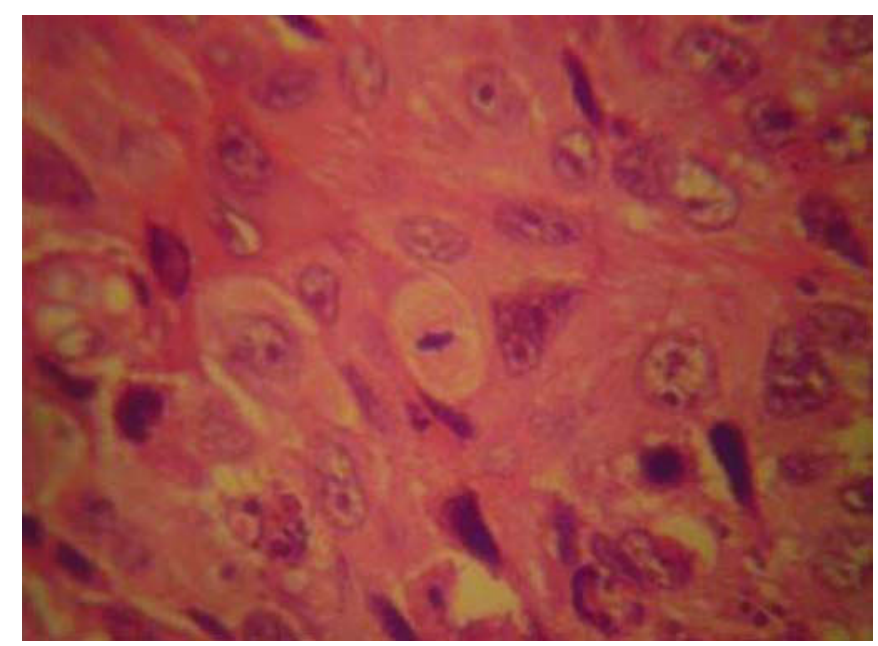

Fig. 9: Abnormal mitoses

- Nuclear hyperchromatism

- Enlarged nucleoli

- Reduction of cellular cohesion

- Keratinization of single cells or cell groups in the prickle cell layer (Kramer IRH et al 1978). ${ }^{13}$

\section{Grading of Epithelial Dysplasia}

- Mild dysplasia: Slight nuclear abnormalities, most marked in the basal third of the epithelial thickness and minimal in the upper layers, where the cell show maturation and stratification. A few, but no abnormal mitoses may be present, usually accompanied by keratosis and chronic inflammation.

- Moderate dysplasia: More marked nuclear abnormalities and nucleoli tend to be present, with changes most marked in the basal two-third of the epithelium, nuclear abnormalities may persist upto the surface, but cell maturation and stratification are evident in the upper layers. Mitoses are present in the parabasal and intermediate layers, but none is abnormal. 
Table 2: Smith-Pindborg criteria

\begin{tabular}{|c|c|c|c|c|}
\hline \multicolumn{2}{|r|}{ Type of change } & \multicolumn{2}{|c|}{ Severity of dysplasia } & \multirow[b]{2}{*}{ Marked } \\
\hline 1. & Drop-shaped rete pegs & None & Slight & \\
\hline 2. & Irregular epithelial stratification & None & Slight & Marked \\
\hline 3. & Keratinization of cells below keratinized layer & None & Slight & Marked \\
\hline 4. & Basal cell hyperplasia & None & Slight & Marked \\
\hline 5. & Loss of intercellular adherence & None & Slight & Marked \\
\hline 6. & Loss of polarity & None & Slight & Marked \\
\hline 7. & Hyperchromatic nuclei & None & Slight & Marked \\
\hline 8. & $\begin{array}{l}\text { Increased nucleocytoplasmic ratio is } \\
\text { basal and prickle-cell layers }\end{array}$ & None & Slight increase & Marked increase \\
\hline 9. & Anisocytosis and anisonucleosis & None & Slight & Marked \\
\hline 10. & Pleomorphic cells and nuclei & None & Slight & Marked \\
\hline 11. & Mitotic activity & & Slight increase & Marked increase \\
\hline 12. & Level of mitotic activity & & Slight & Marked \\
\hline 13. & Presence of bizarre mitoses & None & Slight & Marked \\
\hline
\end{tabular}

- Severe dysplasia: Marked nuclear abnormalities and loss of maturation involve more than two-third of the epithelium, with some stratification of the most superficial layers. Mitoses some of which are abnormal may be present in the upper layers.

\section{Kramer (1980) ${ }^{14-16}$}

This grading system suggests that an epithelium shows dysplasia if it has any two or more of the following features:

- Drop shaped rete pegs: Rete pegs that are wider in the deeper portions than they are more superficially.

- Loss of polarity of the basal cells: Where the basal cells are not perpendicular to the epithelial connective tissue junction, but are at an angle to the junctions.

- Basal cell hyperplasia: The development of basal layer that is several layers thick.

- Loss of epithelial stratification or loss of polarization: Is due to an apparent inability to properly differentiate and mature from basal cells to prickle cells to flattened keratinocytes, thus affecting the regular stratification pattern.

- Cellular pleomorphism or anisocytosis: Variation in the size and shape of the cells.

- Nuclear hyperchromatism: The nuclei in the cells are darkly stained due to increased DNA synthesis.

- Prominent nucleoli: Enlarged, often eosinophilic nucleoli. May stand out like a golf ball.

- Increase in nuclear cytoplasmic ratio: The nucleus enlarges and occupies a greater part of the cell as compared to the cytoplasm (normal ratio is 1:4 to 1:6).

- Cell crowding: Cells appear to be crowded more closely than normal keratinocytes. There is an increase in the number of cells per unit area brought about by basal cell hyperplasia.

- Increased mitosis: Is the increase in frequency of mitotic figures.
- Mitosis in upper layers: Is the spread of mitotic activity to the higher levels of the epithelium.

- Abnormal mitosis: May be defined as mitotic figures found in unusual locations above the basal cell layer, e.g.: Tripolar or star-shaped mitotic figures.

- Loss of cellular adhesion or cohesion: The cells lose their attachment to the neighboring cells, because of faulty or reduced attachment of their desmosomes.

- Intraepithelial keratinization and individual cell keratinization: Is premature production of keratin within the cytoplasm of individual cells or group of cells.

\section{Burkhardt and Maerkar (1981) 17-19}

They used the following characteristics:

- Basal cell hyperplasia

- Loss of basal cell polarity

- Cellular pleomorphism

- An increase in mitotic figures

- Dyskeratosis

- Abnormal and absent epithelial stratification.

Additional indicators for dysplasia were as follows:

- An increase in subepithelial lymphocytes, plasma cells and interepithelial cells (stroma reaction)

- Presence of Candida organisms.

\section{Grading}

They graded dysplastic criteria for classification according to the degree of dysplasia and characteristics of carcinoma in situ as listed in Table 3.

\section{Shafer $(1983)^{14}$}

Shafer listed the criteria for epithelial dysplasia:

- Increased and particularly abnormal mitosis

- Individual cell keratinization

- Epithelial pearls within spinous layer

- Alteration in the nuclear cytoplasmic ratio 
- Loss of polarity

- Large prominent nucleoli

- Dyskaryosis

- Poikilokaryosis

- Basilar hyperplasia.

\section{Grading}

Based on the number of individual histological features and extension of the cytological changes from the basal cell layer and upward epithelial dysplasia has been subdivided into:

- Mild (Grade I): Demonstrates proliferation of atypical or immature basal cells above the parabasal region but not extending beyond the lower third of the epithelium.

- Moderate (Grade II dysplasia): Similar proliferation as in grade I into the middle one-third of the epithelium.

- Severe grades (Grade III): Reserved for abnormal proliferation from the basal layer into the upper third of the epithelium.

\section{Lumermann $\mathrm{H}$ et al (1995) ${ }^{2}$}

They considered the following features as 'minimal' criteria for the diagnosis of oral epithelial dysplasia. The features are:

- Basal cell hyperplasia

- Nuclear enlargement and hyperchromicity

- Drop-shaped rete pegs.

\section{Grading}

The dysplastic changes were graded as:

- Mild epithelial dysplasia: 'Minimal' dysplastic alterations confined to the lower third of the epithelium.

- Moderate epithelial dysplasia: Dysplastic changes seen in upto two-thirds of the thickness of the epithelium.

Table 3: Burkhardt and Maerkar grading system

\begin{tabular}{ll}
\hline Degree & Characteristics \\
\hline Low & - Basal cell hyperplasia \\
& - Basal cell polarity disrupted \\
Medium & - Basal cell hyperplasia \\
& - Loss of basal cell polarity \\
& - Moderate degree of cellular polymorphism \\
& - Slight increase in rate of mitosis \\
& - Occasional dyskeratosis \\
High & - Basal cell hyperplasia \\
& - Basal cell polarity lost \\
& - Marked cellular pleomorphism \\
& - Increase in ratio of mitosis \\
& - Numerous dyskeratosis \\
& - Abnormal epithelial stratification \\
& - Characteristics of high degree dysplasia \\
Ca in situ & more marked \\
& - Epithelial stratification lost \\
& - Stroma not yet invaded \\
\hline
\end{tabular}

- Severe epithelial dysplasia: Dysplastic cells fill more than two-thirds but less than the entire thickness of the epithelium.

- Carcinoma in situ: The entire thickness of the epithelium contains less differentiated basaloid or squamous epithelial cell with enlarged, hyperchromatic nuclei and a variable number of typical and atypical mitotic figures with no invasion into the submucosa.

- Verrucous hyperplasia with dysplasia: The epithelium exhibits considerable thickening with surface papillations, hyperparakeratosis and parakeratin plugging and occasional dysplastic cells confined to the lower one-third of the epithelium. ${ }^{16,19}$

\section{Neville et al (1995) ${ }^{20}$}

Neville et al graded dysplasia as:

- Mild: Hyperchromatic and slightly pleomorphic nuclei are noted in the basal and suprabasal cell layers of stratified squamous epithelium.

- Moderate: Dysplastic changes extend from the basal layer to the midportion of the spinous layer and are characterized by nuclear hyperchromatism, pleomorphism and cellular crowding. Hyperkeratosis on the epithelial cell layer with prominent granular cell layer.

- Severe: Cellular crowding and disordered arrangement throughout most of the epithelial thickness, although slight maturation and flattening of the cells appears to be present at the epithelial surface. Epithelial cells are seen to mature very little as they progress toward the hyperparakeratotic surface.

- Carcinoma in situ: When the entire thickness of the epithelium is involved, the term carcinoma in situ is used. Dysplastic cells extend from the basal layer to the surface of the mucosa (top to bottom change) with no invasion into the underlying connective tissue.

\section{Speight PM et al (1996) ${ }^{21}$}

They considered the thickness (height) to which the cellular and tissue changes may extend as important in grading dysplasia. ${ }^{5,19,22}$

\section{Grading}

According to them:

- Mild forms of dysplasia: Represented recognizable changes limited to the parabasal layers (lower third).

- Moderate dysplasia: Represented recognizable changes extending to middle third.

- Severe dysplasia: Represented as recognizable changes extending to the upper layers. 
- Drawback: Warnakulasuriya $2001^{11}$ commented that there was wide variation in the thickness of the covering epithelium in the oral cavity, which leads to practical difficulties in using this grading system.

\section{Kuffer and Lombardi (2002) ${ }^{23}$}

They felt the choice of clinical rather than histological criteria in the diagnosis and terminology of precancer is the cause of a disorderly mixture of dysplastic and nondysplastic lesions. Therefore, they proposed to dismember the classical 'oral precancerous lesions' to classify all cases which histologically do not show dysplasia into the category of 'risk lesions' (e.g. simple tobacco keratosis) and to place lesions with dysplasia (i.e. already engaged in the process of malignant transformation) into the category of 'precursors' of squamous cell carcinoma (e.g. tobacco keratosis with dysplasia). This 'precursor' term seems to be the most accurate to characterize the limited but already malignant intraepithelial alterations of dysplasia and carcinoma in situ, which herald the onset of an invasive squamous cell carcinoma.

The drawback is that there was considerable difference in potential for transformation between lesions without dysplasia or with mild-moderate dysplasia and those with severe dysplasia, the application of the term 'risk lesion' to lesions without dysplasia which have a 'zero risk' of transformation (e.g. frictional keratosis) was inappropriate.

The use of the term 'precursor of oral squamous cell carcinoma' to dominate dysplastic lesions suggested that they were unequivocally associated with the future development of cancer. On the contrary, as demonstrated by Mincer et al, ${ }^{24}$ $20 \%$ of oral dysplasia regressed and $40 \%$ showed no modification in severity. According to Gupta et at ${ }^{25} 13 \%$ of cases regressed and $40 \%$ showed no modification in severity.

Richard $^{26}$ demonstrated that dysplasia and carcinoma in situ were different aspects of the same disease 'cervical intraepithelial neoplasm(CIN)' and treatment should be same for both. This concept of CIN has now replaced almost completely that of cervical dysplasia. It has been extended with some modification to oral mucosa as 'oral intraepithelial neoplasm(OIN)' and in general as 'squamous intraepithelial neoplasm (SIN)'.19

As for CIN, there are three grades of OIN:

- OIN 1: Mild dysplasia less than one-third involvement of the epithelium

- OIN 2: Moderate dysplasia one-third to two-third involvement of the epithelium

- OIN 3: Severe dysplasia-full involvement or equivalent to carcinoma in situ.

The 'Bethesda classification'18 for cervical pathology includes only two grades:

- Low grade SIN corresponds to CIN 2

- High grade SIN corresponds to CIN2, CIN 3
- Based on this Bethesda classification, the former system with three grades was replaced by a 2-grade system, which helped in better stratifying patients for clinical protocols. Accordingly they chose to report the diagnosis of oral dysplastic lesions as:

- Low grade OIN-including OIN 1 (mild dysplasia) or as

- High grade OIN-including OIN 2 (moderate dysplasia) and OIN 3 (severe dysplasia).

\section{Ljubljana Grading System}

This classification was developed to cater for the special clinical and histological problems of laryngeal abnormalities. ${ }^{3}$ The classification was proposed for grading of epithelial hyperplastic lesions of the larynx, to hyperplastic epithelial lesions arising in the oral cavity.

\section{Grading}

- Simple hyperplasia: A benign hyperplastic process with retention of the normal pattern of the epithelium which is thickened because of an increase prickle cell layer. The cellular components of the basal and parabasal region remain unchanged. There is no cellular atypia.

- Abnormal hyperplasia: A benign augmentation of basal and parabasal layers. This is seen upto $1 \frac{1}{2}$ of the total epithelial thickness. Stratification is fully retained. Nuclei in the cells of the basal and parabasal layers may be moderately enlarged but still maintain a uniform distribution of nuclear chromatin. Small numbers of epithelial cells, less than 5\% are dyskeratotic.

- Atypical hyperplasia or 'risky' epithelium: It demonstrates a recognizable alteration of epithelial cells toward malignancy, but not to such a degree as seen in carcinomatous cells. Stratification is still preserved in the general epithelial structure. The nuclei are enlarged and nuclear contour may be irregular with marked variations in staining intensity. The nuclear cytoplasmic ratio is increased. Mitotic figures are increased and are found within two-third of the epithelium. Civatte bodies (apoptotic cells) may be present.

- Carcinoma in situ: It shows features of carcinoma without invasion. There is loss of stratification throughout the epithelium although 3 to 5 layers of compressed cells may be present on the surface. Marked atypia and mitotic abnormalities are characteristic. Mitotic figures present throughout the epithelium, including its upper one-third and abnormal mitoses are frequently found.

\section{Brothwell DJ et al (2003)}

Brothwell et $\mathrm{al}^{27}$ graded 64 sections of epithelial dysplastic lesions according to 5 point scale routinely utilized at their institution (Faculty of Dentistry, University of Toronto). 


\section{Criteria}

- $0=$ No dysplasia

- 1 = Mild dysplasia: Increased number of cells in the basal and parabasal epithelial regions showing nuclear hyperchromatism and pleomorphism.

- 2 = Moderate dysplasia: Bulbous rete pegs with increased numbers of cells showing nuclear hyperchromatism and pleomorphism, extending to and including the basal, parabasal and prickle cell layer.

- $3=$ Severe dysplasia: Bulbous rete pegs with increased numbers of cells showing nuclear hyperchromatism and pleomorphism through the entire thickness of epithelium.

- 4 = Carcinoma in situ: Markedly atypical changes showing nuclear hyperchromatism and pleomorphism in entire thickness of the epithelium, with the suggestion of early superficial connective tissue invasion, but without convincing evidence.

The advantage is that using this system, and a different method of statistical analysis, the authors proved that intra- and interobserver agreement in grading the dysplastic lesions were consistent and had almost perfect conformity.

\section{WHO System (2005)}

The criteria used for dysplasia are listed in Table 1.

\section{Grading}

On the basis of architecture and cytology ${ }^{28}$

- Hyperplasia: Describes increased cell numbers. This may be in the spinous layer leading to hyperplasia or acanthosis in the basal/parabasal cell layers (progenitor compartment), termed basal cell hyperplasia. Architecture shows regular stratification and there is no cellular atypia.

- Mild dysplasia: Slight nuclear abnormalities, most marked in the basal third of the epithelial thickness. Cells show normal maturation and stratification. A few, but no abnormal mitoses may be present in the parabasal layers.

- Moderate dysplasia: More marked nuclear abnormalities are seen in the basal two-third of the epithelium. Cell maturation and stratification are evident in the upper layers. Mitoses are present in the parabasal and intermediate layers, but none is abnormal.

- Severe dysplasia: Marked nuclear abnormalities involve more than two-thirds of the epithelium. Mitoses, some of which are abnormal, may be present in the upper layers. Maturation and stratification was still seen in most superficial layers.

- Carcinoma in situ: It is defined as 'a lesion in which the full thickness, or almost the full thickness, of squamous epithelium shows the cellular features of carcinoma without stromal invasion.' Requires top-tobottom change with undifferentiated, primitive cells from the basal layer to the topmost layer.

\section{Binary System (2005)}

This system was proposed by Omar Kujan et $\mathrm{al}^{29}$ and considered the lesions under:

High risk lesions (with potential susceptibility for malignant transformation): It was based on observing at least four architectural changes and five cytological changes (WHO criteria 2005).

Low-risk lesions (does not have the potential susceptibility for malignant transformation): It was associated with observation of less than four architectural changes or less than five cytological changes (WHO criteria 2005).

\section{Biomarkers for Dysplasia}

Currently, there is not a substantial body of strong evidence for the use of biomarkers in the progression of oral dysplasia. There is a suggestion from the longitudinal studies that the presence of LOH/A1 at specific loci (3p and 9p), survivin, MMP9 positivity and DNA content (nondiploid) are potential markers for increased risk of progression from oral dysplasia to cancer. ${ }^{30}$ Other markers identified are p53, p73, MMP1, MMP2 and cathepsin L mRNA, but did not predict progression.

\section{CONCLUSION}

Grading of dysplasia continues to be a hotly debated subject. Dysplasia grading is also subjective with inter- and intraobserver variability. Moreover, due to the absence of a consensus, several systems are currently employed. There is no doubt that in future molecular biology discoveries and routine ways of assessing gross genomic aberrations will provide improved ways of assisting diagnosis and prediction of prognosis of oral dysplastic lesions and therefore, a better system for the prediction of cancer progression is needed. Histopathological assessed severity of oral epithelial dysplasia remains the 'gold standard' for the prediction of malignant transformation of precancerous lesions.

\section{REFERENCES}

1. Pindborg JJ, Reichart P, Smith CJ, Van der Waal I. World Health Organization: Histological typing of cancer and precancer of the oral mucosa. Berlin: Springer-Verlag, 1997;21-26

2. Lumermann H, Freedman P, Kerpel S. Oral epithelial dysplasia and the development of invasive squamous cell carcinoma. Oral Surg Oral Med Oral Path Oral Radiol Endod 1995;79:321-29.

3. Warnakulasuriya S, Reibel J, Bouquot J, Dabelstein E. Oral epithelial dysplasia classification systems: Predictive value, utility, weaknesses and scope for improvement. J Oral Path Med 2008;37:127-33. 
4. Califano J, Westra WH, Meininger G, Corio R, Koch WM, Sidransky D. Genetic progression and clonal relationship of recurrent premalignant head and neck lesions. Clin Cancer Res 2000;6:347-52.

5. Scully C, Subdo J, Speight PM. Progress in determining the malignant potential of oral lesions. J Oral Path Med 2003;32:1251-56.

6. Albert B. Molecular biology of the cell (5th ed). Garland Science 2007;223-36

7. Lewin B. Genes IX. (9th ed). Sudbury MA: Jones and Bartlett 2008;813-39.

8. Sharma N, Jagadish VH, Vaibhav T. Epithelial Dysplasia: Different grading system and its applications. J Int Oral Health 2010;2(1):1-8.

9. Smith CJ, Pindborg JJ. Histological grading of oral epithelial atypia by the use of photographic standards. Copenhagen: C. Hamburgers Bogtrykkeri 1969;5-30.

10. Katz HC, Shear M, Altini M. A critical evaluation of epithelial dysplasia in oral mucosal lesions using the Smith-Pindborg method of standardization. J Oral Pathology 1985;14:476-82.

11. Warnakulasuriya S. Histological grading of oral epithelial dysplasia revisited. J Pathol 2001;194:294-97.

12. Bancozy J, Csiba A. Occurrence of epithelial dysplasia in oral leukoplakia: Analysis and follow-up study of 120 cases. Oral Surg Oral Med Oral Pathol 1976;42(6):766-74.

13. Kramer IRH, Lucas RB, El-Labban N, Lister L. A computeraided study on the tissue changes in oral keratosis and Lichen Planus and an analysis of case groupings by subjective and objective criteria. Br J Cancer 1970;24:407-23.

14. Shafer WG, Hine MK, Levy BM, Rajendran R, Sivapathasundharam B. Textbook of oral pathology (6th ed). New Delhi: Elsevier 2009;86-91.

15. Kramer IRH, Lucas RB, El-Labban N, Lister L. The use of discriminant analysis for examining the histological features of oral keratosis and lichen Planus. Br J Cancer 1970;24(3): 673-83.

16. Kramer IRH, Lucas RB, Pindborg JJ, Sobin LH. Definition of leukoplakia and related lesions: An aid to studies on oral precancer. Oral Surg Oral Med Oral Pathol 1978;46(4):518-39.

17. Shklar. Oral cancer. Tokyo: WB Saunders Company 1984;9-19.

18. Soames JV, Southerm JC. Oral pathology. New York: Oxford Medical Publications 1998:138-66.

19. Gnepp DR. Diagnostic surgical pathology of the head and neck. Philadelphia: WB Saunders Company 2001;4-10.

20. Neville BW, et al. Oral and maxillofacial pathology. London: WB Saunders Company 2001:343.

21. Speight PM. Update on oral epithelial dysplasia and progression to cancer. Head Neck Pathol 2007;1:61-67.

22. Cawson RA, Binnie WH, Speight PM, Barrett AW, Wright JM. Luca's pathology of tumors of the oral tissues London: Churchill Livingstone 1998:223-24.

23. Kuffer J, Lombardi S. Reconsideration oral risk lesions. Oral Dis 2002;38:302-07.
24. Mincer HH, et al. Observations on the clinical characteristics of oral lesions showing histological epithelial dysplasia. Oral Surg Oral Med Oral Pathol 1972;3:389-99.

25. Mehta FS, Pindborg JJ, Gupta FC, Daftary DK. Epidemiological and histological study of oral cancer and leukoplakia among 50,915 Indian villagers. Cancer 1969;24:844-61.

26. Richard J, Oliver, MacDonald G, David H. Aspects of cell proliferation in oral epithelial dysplastic lesions. J Oral Pathol Med 2000;29:49-55.

27. Brothwell DJ, Lewis DW, Bradley G, Leong I, Jordan RCK. Observer agreement in the grading of oral epithelial dysplasia. Community Dent Oral Epidemiol 2003;31:300-05.

28. Barnes L, Eveson JW, Reichart P, Sidransky D. World Health Organization Classification of Tumors. Pathology and genetics of head and neck tumors. IARC Press: Lyon 2005;177-79.

29. Kujan O, Richard JO, Khattab A, Stephen A. Evaluation of a new binary system of grading oral epithelial dysplasia for prediction of malignant transformation. Oral Oncology 2006;42(10):987-93.

30. Smith J, Rattay T, McConkey C, Helliwell T, Mehanna H. Biomarkers in dysplasia of the oral Cavity: A systemic review. Oral Oncol 2009;45:647-93.

\section{ABOUT THE AUTHORS}

\section{Varun Rastogi (Corresponding Author)}

Senior Lecturer, Department of Oral Pathology, Kalka Dental College Meerut, Uttar Pradesh, India, e-mail: drvarunrastogi@gmail.com

\section{Naveen Puri}

Professor and Head, Department of Oral Pathology, Kalka Dental College, Meerut, Uttar Pradesh, India

\section{Satyaranjan Mishra}

Reader, Department of Oral Medicine and Radiology, Institute of Dental Sciences, Bhubaneshwar, Odisha, India

\section{Swati Arora}

Senior Lecturer, Department of Oral Pathology, Kalka Dental College Meerut, Uttar Pradesh, India

\section{Geetpriya Kaur}

Senior Lecturer, Department of Oral Pathology, Kalka Dental College Meerut, Uttar Pradesh, India

\section{Lalita Yadav}

Senior Lecturer, Department of Oral Pathology, Kalka Dental College Meerut, Uttar Pradesh, India 